\title{
Resting-state fMRI and developmental systems neuroscience
}

\author{
Lucina Q. Uddin* \\ Department of Psychiatry and Behavioral Sciences, Stanford University School of Medicine, Stanford, CA, USA \\ *Correspondence: lucina@stanford.edu
}

\section{A commentary on}

Maturing thalamo-cortical functional connectivity across development

by Fair, D. A., Bathula, D., Mills, K. L., Dias, T. G., Blythe, M. S., Zhang, D., Snyder, A. Z., Raichle, M. E., Stevens, A. A., Nigg, J. T., Nagel, B. J. (2010). Front. Syst. Neurosci. 4:10. doi: 10.3389/fnsys.2010.00010

Over the past several years, resting-state fMRI (rsfMRI) has emerged as a novel informative method for investigating the development of large-scale functional networks in the human brain. This method, first used to demonstrate coherent spontaneous low-frequency fluctuations in BOLD signal within the adult somatomotor system (Biswal et al., 1995), involves collecting functional imaging data from participants as they lay in the MRI scanner in the "resting state." Participants are typically instructed to fixate on a cross-hair or to keep their eyes closed, and refrain from engaging in any particular cognitive task. This procedure allows for the assessment of resting-state functional connectivity using spontaneous synchronized fluctuations in BOLD signal to determine functional brain networks independent of transient task-induced correlations. The advantages of using rsfMRI in pediatric and clinical neuroscience are that functional brain organization can be examined independent of behavioral performance, and a full dataset can be collected in as little as 5 min (Van Dijk et al., 2009). The resting-state scanning procedure places a minimal cognitive burden on the participant, and requires relatively little time in the scanner compared to task fMRI studies, permitting neuroimaging data collection from very young populations without the need for sedation. For these reasons, developmental cognitive neuroscientists are increasingly incorporating resting-state fMRI into their research programs (see Uddin et al., 2010 for review).

Functional connectivity measures derived from rsfMRI have been used to demonstrate age-related changes in the organization of networks across the whole brain (Fair et al., 2009; Supekar et al., 2009) as well as within specific systems (Kelly et al., 2009). The majority of research efforts have been directed at investigating the development of cortical systems, however cortical-subcortical and in particular thalamo-cortical connectivity has not been as well-studied. Thalamo-cortical changes are thought to occur throughout development (Giedd et al., 1999), yet very few studies have attempted to catalog these changes from childhood through adolescence and into adulthood. To address this, Fair and colleagues applied a novel method to characterize developing thalamo-cortical interactions. Using rsfMRI collected from children (age $8.57 \pm 0.67$ ), adolescents $(13.02 \pm 1.37)$, and adults $(25.33 \pm 3.85)$, the investigators mapped thalamo-cortical interactions in a manner described in a previous publication from their group (Zhang et al., 2008). Briefly, known cortical anatomy was first used to identify functional boundaries in the thalamus. Next, regions of interest (ROIs) encompassing major cortical subdivisions (prefrontal cortex, motor/ premotor, somatosensory, parietal/occipital, and temporal) were identified. The average spontaneous rsfMRI signal generated within each cortical ROI was then correlated with all of the brain voxels within the thalamus. Using a "winner take all" strategy (where the cortical subdivision that correlates strongest with a given voxel "wins"), the thalamus was partitioned into distinct subdivisions. As the authors previously reported, the resulting thalamic subdivisions were in agreement with thalamic nuclear grouping based on postmortem studies of the human brain (Zhang et al., 2008).

The authors replicated their prior work examining thalamo-cortical functional connectivity in adults (Zhang et al., 2008), finding that the parietal-occipital cortical subdivision showed correlations with the lateral and posterior portions of the thalamus (lateral pulvinar, lateral geniculate), the temporal cortical subdivision correlated with medial, inferior, and posterior portions of the thalamus (medial pulvinar, medial geniculate, and medial dorsal nucleus), the prefrontal cortical subdivision showed correlations with dorsal, medial, and anterior portions of the thalamus (medial dorsal and anterior nuclear groups), somatosensory cortical areas correlated with ventral, lateral, and posterior thalamic regions (ventral posterolateral and posteromedial nuclei), and the motor cortex subdivision correlated with lateral and ventral thalamic areas (ventral lateral and ventral anterior nuclei). The greatest developmental differences in thalamo-cortical connectivity were seen between the youngest and oldest age groups. Frontal lobe correlations with the thalamus were found to be weak in childhood and to strengthen by adulthood. Temporal lobe correlations with the thalamus, however, were stronger in children, and weaker in adolescence and adulthood. In addition, premotor-motor and somatosensory cortical subdivisions showed increased connectivity in lateral/inferior portions of the thalamus and decreased connectivity in medial/dorsal portions of the thalamus, while occipital-parietal correlations with the thalamus were relatively unchanged from childhood to adolescence to adulthood (Fair et al., 2010).

It has recently been shown using rsfMRI that children show stronger subcorticocortical connectivity whereas adults show stronger cortico-cortical connectivity (Supekar et al., 2009). The current findings documented by Fair and colleagues provide additional nuanced insights, suggesting that the dynamic process of thalamo-cortical connectivity maturation involves differential strengthening and weakening of specific connections between cortical regions and subdivisions of the thalamus. This specificity, and deviations from this pattern seen in typical development, may be of consequence both functionally and behaviorally. The investigation of neurodevelopmental disorders using rsfMRI is in its infancy, but there are already hints that the method can be used to reveal subcortico-cortical connectivity alterations in clinical populations (Di Martino et al., 2011). 
The study by Fair and colleagues reviewed here is an elegant example of the promise of rsfMRI in addressing important research questions in developmental systems neuroscience. Future directions for the field include longitudinal studies that allow for tracking the development of subcortico-cortical and cortico-cortical connectivity within individuals. Such studies will provide additional insights into typical human brain development, as well as potentially inform research studying the neural bases of developmental disorders such as attention-deficit/hyperactivity disorder and autism spectrum disorders.

\section{REFERENCES}

Biswal, B., Yetkin, F. Z., Haughton, V. M., and Hyde, J. S. (1995). Functional connectivity in the motor cortex of resting human brain using echo-planar MRI. Magn. Reson. Med. 34, 537-541.

Di Martino, A., Kelly, C., Grzadzinski, R., Zuo, X. N., Mennes, M., Mairena, M. A., Lord, C., Castellanos,
F. X., and Milham, M. P. (2011). Aberrant striatal functional connectivity in children with autism. Biol. Psychiatry (in press).

Fair, D. A., Bathula, D., Mills, K. L., Dias, T. G., Blythe, M. S., Zhang, D., Snyder, A. Z., Raichle, M. E., Stevens, A. A., Nigg, J. T., and Nagel, B. J. (2010). Maturing thalamocortical functional connectivity across development. Front. Syst. Neurosci. 4:10. doi: 10.3389/ fnsys.2010.00010

Fair, D. A., Cohen, A. L., Power, J. D., Dosenbach, N. U. Church, J.A., Miezin, F.M.,Schlaggar, B.L., and Petersen, S.E. (2009). Functional brain networks develop from a "local to distributed" organization. PLoS Comput. Biol. 5, e1000381. doi: 10.1371/journal.pcbi.1000381

Giedd, J. N., Blumenthal, J., Jeffries, N. O., Castellanos, F. X., Liu, H., Zijdenbos, A., Paus, T., Evans, A. C., and Rapoport, J. L. (1999). Brain development during childhood and adolescence: a longitudinal MRI study. Nat. Neurosci. 2, 861-863.

Kelly, A. M., Di Martino, A., Uddin, L. Q., Shehzad, Z. Gee, D. G., Reiss, P. T., Margulies, D. S., Castellanos, F. X., and Milham, M. P. (2009). Development of anterior cingulate functional connectivity from late childhood to early adulthood. Cereb. Cortex 19, 640-657.

Supekar, K., Musen, M., and Menon, V. (2009). Development of large-scale functional brain networks in children. PLoS Biol. 7, e1000157. doi: 10.1371/journal.pbio.1000157

Uddin, L. Q., Supekar, K., and Menon, V. (2010). Typical and atypical development of functional human brain networks: insights from resting-state FMRI. Front. Syst. Neurosci. 4:21. doi: 10.3389/fnsys.2010.00021

Van Dijk, K. R., Hedden, T., Venkataraman, A., Evans, K. C., Lazar, S. W., and Buckner, R. L. (2009). Intrinsic functional connectivity as a tool for human connectomics: theory, properties, and optimization. J. Neurophysiol. 103, 297-321.

Zhang, D., Snyder, A. Z., Fox, M. D., Sansbury, M. W., Shimony, J. S., and Raichle, M. E. (2008). Intrinsic functional relations between human cerebral cortex and thalamus. J. Neurophysiol. 100, 1740-1748.

Received: 12 January 2011; accepted: 24 January 2011; published online: 23 February 2011.

Citation: Uddin LQ (2011) Resting-state fMRI and developmental systems neuroscience. Front. Neurosci. 5:14. doi: 10.3389/fnins.2011.00014

Copyright (C) 2011 Uddin. This is an open-access article subject to an exclusive license agreement between the authors and Frontiers Media SA, which permits unrestricted use, distribution, and reproduction in any medium, provided the original authors and source are credited. 